\title{
A New 3-Phase Design Exploration Methodology for Video Processor Design
}

\author{
Wing-Yee Lo, Daniel P.K. Lun and Wan-Chi Siu \\ Centre for Signal Processing \\ Department of Electronic and Information Engineering \\ The Hong Kong Polytechnic University, Hong Kong \\ Email: \{03723060g, enpklun, enwcsiu\}@polyu.edu.hk
}

\begin{abstract}
When making video processor design, conventional design exploration methodologies take extremely long time in parameter optimization but the final design may not necessarily meet the application requirements since the architecture cannot deviate too much from the initial design. To speed up the design process, statistical performance models were used to guide the simulation; however their accuracy is questionable. In this paper, a new 3-phase design exploration methodology for video processor is proposed. It makes use of an almost cycle-accurate performance model to provide information for refining the processor architecture. It can derive the optimal architecture in a much shorter period of time than the conventional methods. We successfully implemented a few video coding/decoding applications on the video processor derived from the proposed methodology. Simulation results show that it outperforms other video processors in both cost and performance perspectives.
\end{abstract}

\section{INTRODUCTION}

Design exploration is an architectural optimization methodology that has been adopted in processor design for years. The idea is to develop a software model with configurable structures to simulate the architecture of the target processor. The performance of the architecture can then be analyzed with different parameter settings so as to find out the optimal architecture design. The traced based approaches have been used traditionally for this purpose [1]-[2]. They use very complex simulation models that take extremely long time for the simulation process in order to get the optimal architecture. To improve the efficiency of the design process, various approaches were proposed such as reducing the benchmark program suite size [3], using trace sampling to reduce the run time [4]-[5], varying only the parameters that have maximum impact [6], and using intelligent optimization method such as the genetic algorithm for architecture optimization [7]. These traditional approaches often require good initial processor architecture and use heuristic searching algorithms to adjust only a few pre-defined parameters to finetune the architecture design. Such methodology can only allow limited architectural variations controlled by the predefined parameters. The optimized architecture may not necessarily meet the application requirements since the

This work is partially supported by the Hong Kong Polytechnic University under grant no 1-BB9B. Most of the research work and implementation development were done in the Hong Kong Applied Science and Technology Research Institute (ASTRI) and Beijing SimpLight Nanoelectroinics Ltd. architecture is limited by the initial architecture. Besides, it takes time to derive good initial architecture which cannot fulfill the fast time-to-market need of the industry.

Another design exploration methodology is the statistical simulation modeling [8]-[9]. This methodology first generates a synthetic instruction trace based on statistical profiling. The instruction trace is then fed into a trace-driven simulator along with the statistics of branch prediction accuracy, cache miss rate, pipeline stall rate and instructions issued per cycle (IPC), etc. The advantage of this methodology is that the simulation time is very short. However, they are in general not accurate.

In this paper, we propose a new 3-phase design exploration methodology and apply it to video processor design. Instead of presetting the initial video processor architecture and searching blindly on all combinations of parameter settings, the proposed methodology allows modifications to the baseline architecture based on the outputs of a performance model. Unlike the statistical model, the performance model used is hardware-friendly and almost cycle-accurate. The proposed design exploration methodology consists of 3 design phases. It starts with a baseline video processor architecture derived from a few basic design criteria. Based on the outputs of the performance model, major architectural changes are made to the baseline architecture to reduce the performance overheads. Finally the design parameters are fine-tuned based on the performance model to achieve an optimal design. Comparing with the conventional design exploration methodology, the proposed approach requires a much shorter design time and allows a higher degree of freedom in selecting the best design parameters. The proposed methodology was adopted in the design of a video processor. It leads to a substantial improvement over the original design and allows the final video processor to be one of the best in the literature measured in terms of cost and efficiency.

\section{Proposed Desgin Exploration Methodology}

The proposed design exploration methodology consists of 3 phases (see Figure 1) which are described as follows:

\section{A. Phase I: Baseline Architecture Development}

The first phase starts with a baseline video processor architecture (see Figure 2) designed with the goal of maximizing the degree of data level parallelism (DLP), instruction level parallelism (ILP) and thread level 


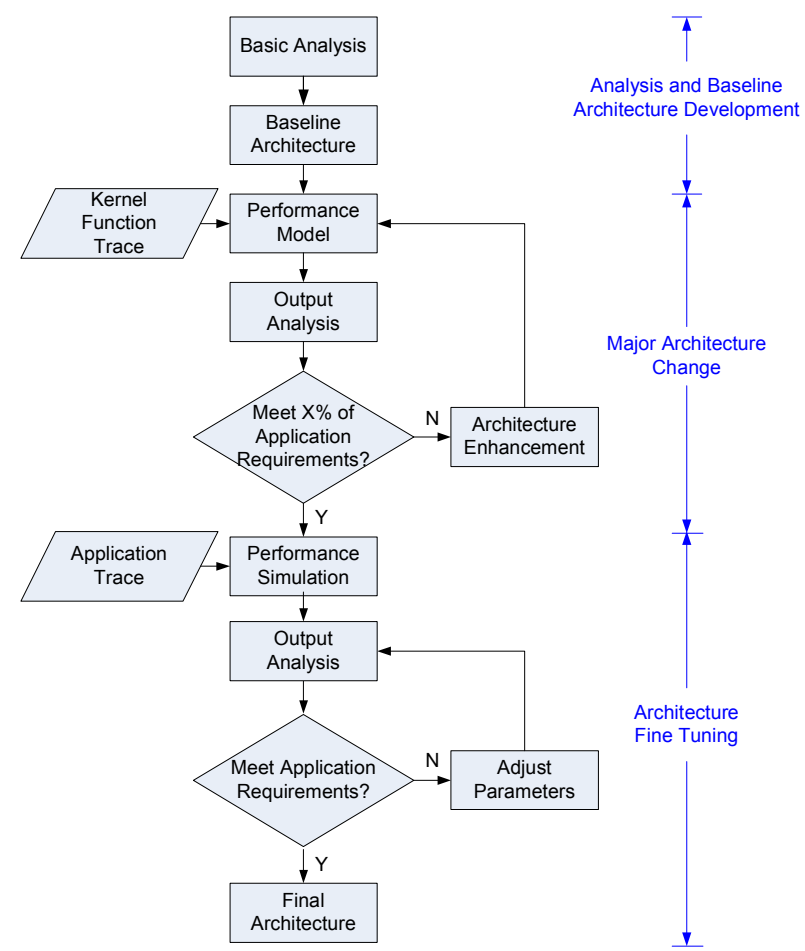

Figure 1. Proposed 3-phase design exploration methodology.

parallelism (TLP). To maximizing the degree of DLP, we equipped the baseline architecture with our recently proposed parallel memory architecture and the configurable SIMD (CSIMD) structure enabled by the CSIMD look-up table (CSLUT) [10]. The ILP is achieved by dynamically issuing a maximum of 4 instructions per cycle using an intelligent instruction fetch and dispatch unit. The TLP is enabled by adopting the multithreading architecture, which supports the execution of a maximum of 2 threads in parallel. The design of this baseline architecture involves detailed analysis of the kernel operations of video coding and decoding. Due to page limitation, they are not detailed in this paper.

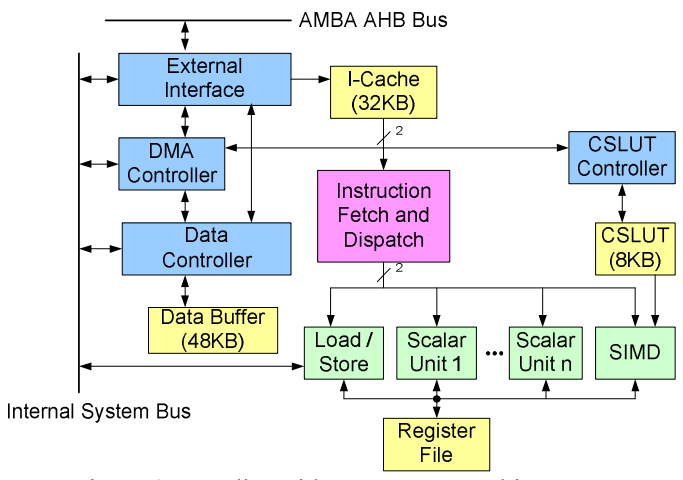

Figure 2. Baseline video processor architecture.

\section{B. Phase II: Major Architecture Changes Using Performance Model}

In the second phase, major architecture modifications are performed on the baseline architecture based on the outputs of a performance model, which is a software system simulating the operation of the target processor. The structure of the performance model is very hardware-like and follows the multi-pipeline stage structure of the baseline video processor. There are five pipeline stages in the video processor. They are instruction fetch (FE), instruction issue (IS), register file data retrieval (RF), execution (EX) and result write back (WB). The performance model does not care about the implementation details except it has to ensure the task assigned in each pipeline stage has sufficient time to accomplish. Major modifications on the baseline architecture are performed based on the outputs of the performance model, which include the architecture performance metrics such as instruction per cycle (IPC) as shown in the instruction issue log, profiling data and statistical data. To save the design exploration time, application kernel functions are used as input trace since the kernel functions can contribute up to more than $75 \%$ of the execution time of the whole application. The input trace is generated by the compiler and software scheduler. It is basically the instruction sequence with meta data that are useful for performance analysis. The output files generated by the performance model are analyzed to see where the performance bottlenecks of the baseline architecture come from. New features are brought up to reduce the performance bottlenecks. Then the performance model, compiler, ISA and/or software scheduler are modified to include the new features and generate another round of performance model outputs. When most application requirements are satisfied, the video processor architecture can be fine-tuned in phase 3 . Figure 3 shows the final performance model after the abovementioned iteration process. The major architectural changes that have been made will be described in next section.

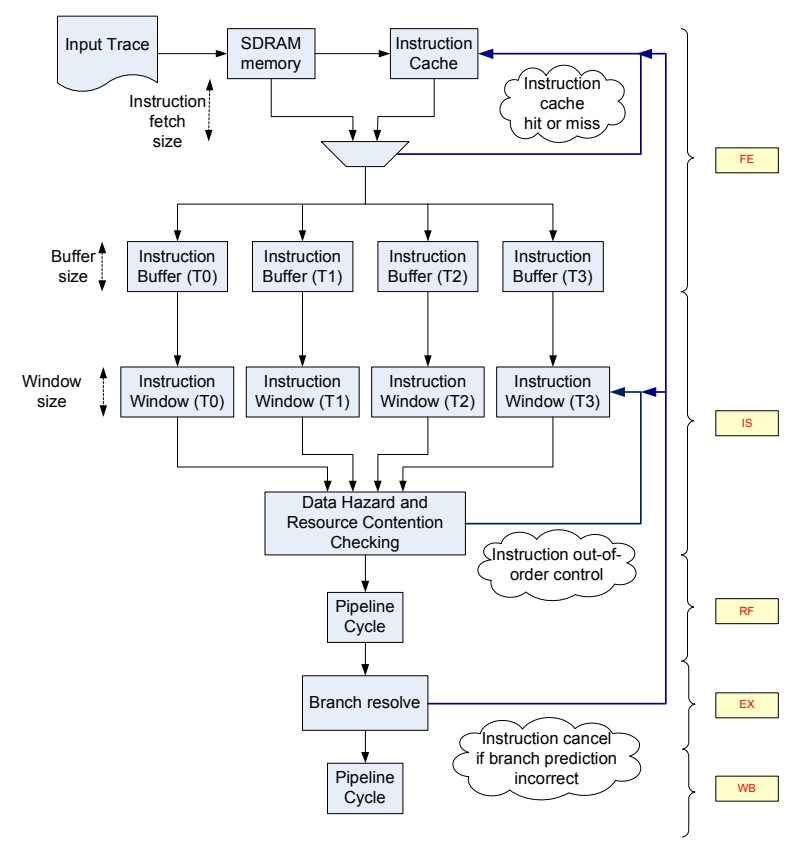

Figure 3. Performance model architecture.

\section{Phase III: Architecture Fine Tuning}

In the last design exploration phase, the processor architecture is fine-tuned. In this phase, the application trace is input to the performance model for the adjustment of different 
parameters to achieve an optimal design. The performance model in fact allows a wide range of parameters to be configured, which include

- Instruction cache organization: cache size, tag size, number of associative sets, etc.

- Number of instructions fetched in one cycle.

- Instruction buffer size.

- Instruction window size (number of instructions from each thread to be selected for dispatch).

- Number of instructions to be issued.

- Multi-functional execution unit.

- Branch prediction algorithm.

- Issue/fetch policy: round-robin, priority order, etc.

- Bypass buses: bypass data between pipeline stages should be supported.

- Number of IO ports in different memory units.

- Out-of-order issue: All or some instructions can be issued out-of-order.

The parameters will be refined until all application requirements are satisfied.

\section{RESULTS}

By using the proposed design exploration methodology, several performance bottlenecks of the baseline architecture were identified. A brief account of these bottlenecks, the architectural changes that have been made to deal with them, and the resulting improvements are given as follows:

1) Multithreading: The first major architecture change is the support of quad-thread from dual-thread. During design exploration, it was observed that the IPC is increased by $21.92 \%$ when the kernel functions are partitioned to execute in 2 threads. While further splitting the execution to more threads should be advantageous, it is desirable if it can be achieved automatically so as to reduce the programming effort of users. A software scheduler was developed for such purpose. The improvement was verified by the performance model that, when using the software scheduler to achieve quad-thread execution, an overall IPC improvement of $39.48 \%$ can be achieved compared with using only single thread.

2) Execution units: There are two architectural changes related to the execution units. The first one is the addition of one more Arithmetic Login Unit (ALU). It stems from the profiling results generated from the performance model that significantly more instructions are executed by the ALU than other execution units. Another change is the replacement of the Multiply-and-Accumulate (MAC) units with normal multipler (MULT) and adder (ADD). It was noticed during design exploration that the lengthy MAC pipeline introduces many pipeline bubbles. Replacing it with normal MULT and ADD pipelines reduce the pipeline length and also the possibility of pipeline bubbles. Besides, it also reduces the branch mis-prediction latency due to the shortened pipeline. The performance model verified that the IPC improvement after the abovementioned modifications is $70.37 \%$.
3) Processor pipeline: The initial video processor supports pipelines of different lengths in various execution units. The instruction issue log generated by the performance model shows that pipelines of irregular length can introduce long execution latency similar to the MAC execution pipeline mentioned above. After going through several rounds of refinement, the final video processor has unified pipeline structure. The performance model showed that this architectural change can lead to a $25.12 \%$ IPC improvement.

4) Micro opcode instruction out-of-order issue: To reduce application program size, the video processor supports macro instructions. A macro instruction consists of a sequence of micro opcode instructions. An example is the block based Sum of Absolute Difference (SAD) instruction which is commonly used in H.264 video coding. Due to the long instuction sequence, it is inevitable for these SIMD macro instructions to have pipeline bubbles. It has large performace impact since the most frequent and performance critical operations are grouped into macro instructions. To solve the problem, we modified the baseline architecture to allow the micro opcode instructions in consecutive independent macro instructions to be issued out of order. It effectively fills up the pipeline bubbles. To fully utilize this feature, the software scheduler was refined to put more independent macro instructions together. The performance model showed that the IPC can be improved by $22.04 \%$.

5) Dynamic load balancing: It was noticed during design exploration that the loading between threads is never balanced due to the picture contents and other reasons. The final video processor architecture supports dynamic load balancing. It provides an instruction for the programmer to indicate any function task to be executed by current thread or another thread, depending on which thread finishes the specified functions first. With this support, the performance model showed that a $15.12 \%$ IPC improvement can be achieved.

\section{PERFORMANCE COMPARISON}

To illustrate the effectiveness of the proposed design exploration methodology and the accuracy of the performance model in predicting the true performance of the processor, we ported several video applications to the final optimized video processor and compared its performance with other video processors in the literature. The final optimized processor is implemented with TSMC $0.13 \mu \mathrm{m}$ technology. The die area is about $8 \mathrm{~mm}^{2}$ including $32 \mathrm{~KB}$ instruction cache, $48 \mathrm{~KB}$ internal SRAM memory and 8KB LUT memory. We show in TABLE I a comparison of the processor frequency and the die area of different video processors designed for encoding or decoding digital video in H.264 format. It shows that the final optimized video processor outperforms others in terms of cost (die area) and performance (frequency). For instance when comparing with [11] and [12], the final optimized video processor works at a much lower frequency to achieve the same frame rate when performing H.264 video encoding. When comparing with [13] and [14], the die area of the final optimized video processor is much smaller to achieve a similar performance in H.264 video decoding. Note that the die area of the design in 
[13] and [14] is our estimation. [13] states that it needs 6 EMC clusters to perform $\mathrm{H} .264$ decoding at $30 \mathrm{fps}$ and D1 resolution using Xilinx Virtex-4 XC4VLX200 FPGA. One EMC cluster consists of two processors, i.e. EMC-0 and EMC-1, which use 23,276 LUT and 12,535 LUT resources in XC4VLX200. Based on the Xilinx Gate Count Capacity Metrics Application Note, the gate count of one LUT ranges from 6-12 gates. We take 8 gates for calculation. Six EMC clusters have about $(23,276+12,535) \times 6=214,866$ LUT which is about $(214,866 \times 8)=1,718,928$ gates. As a well-known rule in ASIC design, one gate count is equivalent to one 2-input NAND gate. A typical drive strength of $\mathrm{x} 2$ 2-input NAND gate is about $0.000008487 \mathrm{~mm}^{2}$ in TSMC $0.13 \mu \mathrm{m}$ technology. Therefore, the estimated equivalent die area of the video processor is about $(1,718,928 \times 0.000008487)=14.59 \mathrm{~mm}^{2}$. In [14], it mentions that the die area of 8 MPE processors is $(5.06 \times 5.06)=25.60 \mathrm{~mm}^{2}$ in $65 \mathrm{~nm}$ technology. The chip micrograph diagram in the reference shows that the area of 8 MPE processors consumes $2 / 3$ of the whole chip. The reminding $1 / 3$ area of the chip is L2 cache memory and L2 cache controller. To decode a VGA picture at $30 \mathrm{fps}$, it needs 3 MPE processors running at $333 \mathrm{MHz}$. Hence the estimated die area is $(25.60 \times(1 / 3+2 / 3 \times 3 / 8))=14.94 \mathrm{~mm}^{2}$. The " $1 / 3$ " in the equation is the area of the L2 cache memory and its controller. The " $2 / 3 \times 3 / 8$ " is the area of 3 MPE processors. The die area of a chip in $65 \mathrm{~nm}$ technology is roughly increased by $3-4$ times when the same logic is mapped to $0.13 \mu \mathrm{m}$ technology. It is commonly used in the ASIC design industry. If so, the video processor in [14] is much larger than the final optimized video processor. Besides, it needs to work at a much higher frequency to meet the application requirements.

\section{SUMMARY}

In this paper, we have proposed a new 3-phase design exploration methodology and applied to video processor design. The methodology starts with a baseline video processor architecture. Based on the outputs of an almost cycle-accurate performance model, the baseline architecture, as well as the compiler, ISA and software scheduler can be refined along the design exploration process. The architecture is then fine-tuned by further adjusting different design parameters. The final optimized video processor showed a substantial improvement over other video processors in the literature in terms of cost and efficiency. It illustrated the effectiveness of the proposed design exploration methodology and the accuracy of the performance model in predicting the true performance of the final video processor.

\section{REFERENCES}

[1] P. Bose and T.M. Conte, "Performance analysis and its impact on design," IEEE Computer, vol. 31, issue 5, pp. 41-49, May 1998.

[2] P. Bose, "Performance evaluation and validation of microprocessors," in Proc. Int. Conf. Meusurement und Modeling of Computer Systems (SlGMETRICS'99), pp. 226-227, May 1999.

[3] A.J.K. Osowski, J. Flynn, N. Meares, and D.J. Lilja, "Adapting the SPEC 2000 benchmark Suite for Simulation-Based Computer Architecture Researh," in Proc. Workshop on Workload Characterization, Int. Conf. Computer Design, Austin, TX, Sept. 2000.

[4] T.M. Conte, M.A. Hirsch, and K.N. Menezes, "Reducing State Loss for Effective Trace Sampling of Superscalar Processors," in Proc., IEEE Int. Conf. Computer Design: VLSI in Computers and Processors, pp. 468-477, Oct. 1996.

[5] V.S. Iyengar and L.H. Trevillyan, "Evaluation and Generation of Reduced Traces for Benchmarks," Technical Report RC20610, IBM, Oct. 1996.

[6] V. Zyuban and P. Kogge, "Optimization of High Performance Superscalar Architectures for Energy Efficiency", in Proc. IEEE Int. Sym. Low Power Electronics and Design, pp. 84-89, 2000.

[7] M. Olivieri, "A Genetic Approach to the Design Space Exploration of Superscalar Microprocessor Architectures", in Proc. IEEE Int. Sym. Circuits and Systems, vol. 5, pp. 69-72, May 2001.

[8] C. Hsieh and M. Pedram, "Micro-Processor Power Estimation Using Profile-Driven Program Synthesis," IEEE Trans. Computer-Aided Design of Integrated Circuits and Systems, vol. 17, issue 11, pp. 10801089, Nov. 1998.

[9] M. Oskin, E T. Chong, and M. Farrens. "HLS: Combining Statistical and Symbolic Simulation to Guide Microprocessor Design," in Proc. 27th IEEE Int. Sym. Computer Architecture, pp. 71-82, Jun. 2000.

[10] W.Y. Lo, D.P.K. Lun, W.C. Siu, W. Wang, and J. Song, "Improved SIMD Architecture for High Performance Video Processors", IEEE Trans. Circuits and Systems for Video Tech., to be published in 2011.

[11] T.R. Jacobs, V.A. Chouliaras and D.J. Mulvaney, "Thread-Parallel MPEG-2, MPEG-4 and H.264 Video Encoders for SoC MultiProcessor Architectures," IEEE Trans. Consumer Electronics, vol. 52, iss. 1, pp. 269-275, Mar. 2006.

[12] W.C. $\mathrm{Ku}$, S.H. Chou, J.C. Chu, C.L. Liu, T.F. Chen, J.I. Guo, J.S. Wang, "VisoMT: A Collaborative Multithreading Multicore Processor for Multimedia Applications with a Fast Data Switching Mechanism," IEEE Trans. Circuits and Systems for Video Tech., vol. 19, no. 11, pp. 1633-1645, Nov. 2009.

[13] J.Y. Lee, J.J. Lee and S.M. Park, "Multi-Core Platform for an efficient H.264 and VC-1 Video Decoding Based on Macroblock Row-Level Parallelism", IET Circuits Devices and System, vol. 4, issue 2, 2010, pp. 147-158.

[14] T. Mori, Y. Ueda, T. Terazawa, M. Sroka, T. Fujita, T. Kodaka, T. Mori, K. Morita, H. Arakida, T. Miura, Y. Okuda, T. Kizu and Y. Tsuboi, "A Power, Performance Scalable Eight-Core Media Processor for Mobile Multimedia Applications", IEEE Journal of Solid-State Circuits, vol. 44, no. 11, pp. 2957-2965, Nov. 2009.

TABLE I. COST AND EFFICIENCY COMPARISONS WITH OTHER VIDEO PROCESSORS.

\begin{tabular}{|c|c|c|c|c|c|c|c|}
\hline \multirow[b]{2}{*}{ Processor } & \multicolumn{2}{|c|}{ Cost } & \multicolumn{4}{|c|}{ Performance } & \multirow[t]{2}{*}{ Remarks } \\
\hline & $\begin{array}{c}\text { Technology } \\
(\mu \mathrm{m})\end{array}$ & $\begin{array}{c}\text { Die Area } \\
\left(\mathrm{mm}^{2}\right)\end{array}$ & $\begin{array}{l}\text { Frequency } \\
(\mathrm{MHz})\end{array}$ & Resolution & $\begin{array}{c}\text { Frame } \\
\text { Rate (fps) }\end{array}$ & Application & \\
\hline \multirow{3}{*}{$\begin{array}{c}\text { Final } \\
\text { optimized } \\
\text { processor }\end{array}$} & \multirow{3}{*}{0.13} & \multirow{3}{*}{8} & 90 & VGA & \multirow{3}{*}{25} & \multirow{2}{*}{ H.264 Decode } & \\
\hline & & & 120 & D1 & & & \\
\hline & & & 90 & CIF & & H.264 Encode & \\
\hline$[11]$ & 0.13 & 7.45 & 170 & $\mathrm{CIF}$ & 25 & H.264 Encode & Die area $=2.73 \times 2.73$ \\
\hline [12] & 0.13 & 32.24 & 180 & $\mathrm{CIF}$ & 33 & H.264 Encode & 1 VisoMT die area: $4.02 \times 4.01$, needs 2 VisoMT \\
\hline$[13]$ & 0.13 & 14.59 & & D1 & 30 & H.264 Decode & $\begin{array}{c}1 \text { EMC clustor: } 23276+12535 \text { LUTs in Xilinx Virtex } 4 \\
\text { XC4VLX200 FPGA, needs } 6 \text { EMC clusters }\end{array}$ \\
\hline [14] & 0.065 & 14.94 & 333 & VGA & 30 & H.264 Decode & $8 \mathrm{MPE}$ is $5.06 \times 5.06$, needs 3 MPE: $\sim(5.06 \times 5.06)^{*}(1 / 3+2 / 3 * 3 / 8)$ \\
\hline
\end{tabular}

